\title{
Reflexiones sobre enfermería desde la biopolítica: relaciones de poder y cuidado
}

Reflection from the biopolitic perspective: power relations and health-care

\section{Reflexões sobre a enfermagem desde a biopolítica: relações de poder e cuidado}

\author{
Claudia Andrea Collado Quezada Correo y Silvana Castillo Parra Correo \\ Departamento de Enfermería, Facultad de Medicina, Universidad de Chile. Chile. \\ Cómo citar este artículo en edición digital: Collado Quezada, C.A., y Castillo Parra Correo, S. (2017). Reflexiones sobre enfer- \\ mería desde la biopolítica: relaciones de poder y cuidado. Cultura de los Cuidados (Edición digital), 21(47). \\ Recuperado de http://dx.doi.org/10.14198/cuid.2017.47.03 \\ Correspondencia: Departamento de Enfermería. Facultad de Medicina. \\ Av. Independencia 1027, Independencia, Santiago (Chile) \\ Correo electrónico: ccollado@med.uchile.cl \\ Recibido: 11/03/2016; Aceptado: 05/09/2016
}

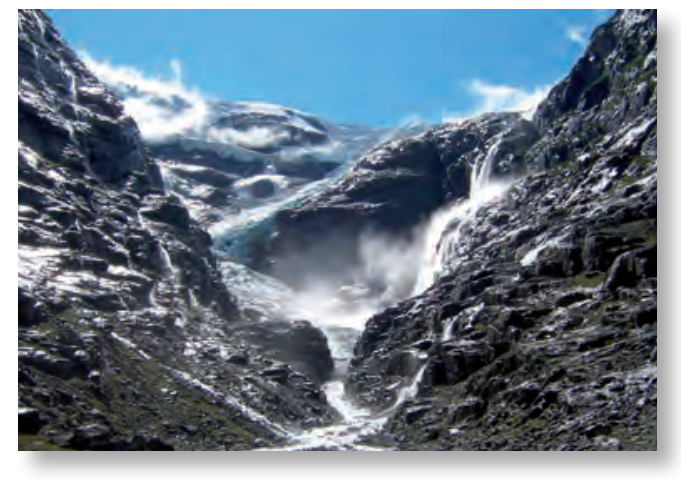

ABSTRACT

This essay invites to a reflection, from the Foucauldian perspective, about the viewpoint of nursing-care delivery and teaching to future professionals, as well as the analysis of the relations of power in nursing and the link with people "caring objects" from a bioethical point of view. The methodology includes texts analysis from a Foucauldian perspective in health, a retrospective view about the experiences of professionals nurses and the installation of mechanism of power in nursing. Sources related to nursing history and the professional formation with emphasis on professor/student and nurse/patient relationship were analyzed.
Bioethical aspects and professional contexts were compared.

Currently nurses have normalized the existence of control mechanism within the direct attention of people and their care, which is transferred to the formation of nurses. Nowadays, nursing thoughts have been expanded, become independent and adapted to different contexts. This invites to use a broader perspective to observe the purpose of the discipline and the profession of nursing, using an introspective view that allows to understand that the nursing practice perpetuates subordination and power in both direct care teams and work teams.

Key words: Nursing, biopolitics, nursing care and discipline.

\section{RESUMO}

Este tema tem como objetivos reflexionar desde a perspectiva foucaultiana, o cuidado que se entrega e se ensina a os futuros professionais e analizar as relações de poder em o ámbito da enfermería e o vínculo com as pessoas "objetos de cuidado" desde um en- 
foque bioético. A metodología incorpora um análises de textos desde a perspectiva foucaultiana em saúde, uma olhada retrospectiva sobre experiências professionais e a instalação de dispositivos de poder em enfermería. Se analizam fontes relacionadas com a história da enfermaría e da formação professional com énfases em as relacões professores/estudantes e enfermeros/pacientes. Se contrastam aspectos bioéticos e contextos professionais.

A enfermería ha normalizado a instalação de lógicas de controle em a atenção direta de pessoas e em o cuidado, do qual se transfere a formação de enfermeiras e enfermeiros. Atualmente, o pensamento de enfermaría se ha ampliado, independizado e adaptado a os contextos, o que convidam a visualizar desde uma perspectiva mais ampla o proposto de que fazer disciplinar e professional, abrindo uma nova forma de observar, a introspectiva que permita compreender, que o exercício da enfermería desde o cotidiano, perpetue lógicas de subordinação e poder tanto em a atenção direta como em as equipes de trabalhos.

Palavras chaves: Enfermaría, biopolítica, cuidado, disciplina.

\section{RESUMEN}

Este ensayo tiene como objetivos reflexionar desde la perspectiva foucaultiana, el cuidado que se entrega y se enseña a los futuros profesionales y analizar las relaciones de poder en el ámbito de la enfermería y el vínculo con las personas "objetos de cuidado" desde un enfoque bioético. La metodología incorpora un análisis de textos desde la perspectiva foucaultiana en salud, una mirada retrospectiva sobre experiencias profesionales y la instalación de dispositivos de poder en enfermería. Se analizan fuentes relacionadas con la historia de enfermería y la formación profesional con én- fasis en las relaciones profesores/estudiantes y enfermeros/pacientes. Se contrastan aspectos bioéticos y contextos profesionales.

La enfermería ha normalizado la instalación de lógicas de control en la atención directa de personas y en el cuidado, lo cual además se transfiere a la formación de enfermeras y enfermeros. Actualmente, el pensamiento de enfermería se ha ampliado, independizado y adaptado a los contextos, lo que invita a visualizar desde una perspectiva más amplia el propósito del quehacer disciplinar y profesional, abriendo una mirada introspectiva que permita comprender, que el ejercicio de la enfermería desde lo cotidiano, perpetúa lógicas de subordinación y poder tanto en la atención directa como en los equipos de trabajo.

Palabras claves: Enfermería, biopolítica, cuidado, disciplina.

\section{INTRODUCCIÓN}

Siendo la enfermería una disciplina en creciente desarrollo, surge la necesidad de reflexionar en torno a cuestiones vinculadas con el cuidado y la biopolítica, entendida esta última como "designar lo que hace entrar a la vida y sus mecanismos en el dominio de los cálculos explícitos y convierte al poder-saber en un agente de transformación de la vida humana" (Foucault, 1977: 85), la cual permea el ámbito de la enfermería en silencio con la comodidad de no ser visualizado. Atrapados en la praxis, enfermeras/os permanecen con una especie de ceguera beneficente, disfrazada del rol de empoderar a las personas que atienden desde el fortalecimiento de la autonomía, mientras sigan las "indicaciones" que les dan para que se hagan "cargo de su propia salud". Esto lleva a divagar en una especie de impulso liberador emanado desde la reflexión y, sin embargo, la 
praxis sigue atravesada por la subordinación y la historia que se construyó, y que "disciplinó" hasta lograr el cuerpo de conocimientos ordenado y confiable que permite mirar el mundo y sus fenómenos desde la ciencia del cuidado.

Se ha partido de los siguientes objetivos:

1) Reflexionar desde la perspectiva foucaultiana, sobre el cuidado que se entrega y se enseña a los futuros profesionales.

2) Analizar las relaciones de poder en el ámbito de la enfermería y el vínculo con las personas "objetos de cuidado" desde un enfoque bioético.

\section{METODOLOGÍA}

Este trabajo fue desarrollado en el marco del curso electivo "De la biopolítica a la bioética. Consideraciones críticas acerca del dispositivo biomédico moderno" del Programa de Magíster en Bioética de la Universidad de Chile, a cargo del Profesor Raúl Villarroel. En una primera instancia se realizó un análisis de textos relacionados con la perspectiva foucaultiana en el ámbito de la salud, además de una mirada retrospectiva sobre experiencias profesionales que evidenciaran la instalación de dispositivos de poder en la enfermería incorporando elementos del panóptico. A continuación se procedió a analizar artículos y libros relacionados con la historia de la enfermería y la formación de profesionales con especial atención a las relaciones establecidas en las diadas profesores/estudiantes y enfermeros/pacientes. Por último, se desarrolla una contrastación entre aspectos bioéticos y los contextos profesionales en enfermería antes descritos.

\section{DESARROLLO DEL TEMA}

No hay duda que la enfermería, desde la construcción profesional y disciplinar en las últimas cinco décadas, ha tenido un avance significativo en cuanto a la construcción de saberes propios, manifestado claramente en la instalación de ella en la academia como disciplina. En Chile, existen en la actualidad normas que respaldan el actuar del profesional en cuanto a la gestión del cuidado "entendida como el ejercicio profesional de la enfermera sustentada en su disciplina, la ciencia de cuidar, se define como la aplicación de un juicio profesional en la planificación, organización, motivación y control de la provisión de los cuidados, oportunos, seguros e integrales, que aseguren la continuidad de la atención y se sustenten en las políticas y lineamientos estratégicos de la institución” (MINSAL, 2009).

Lo anterior, hace pensar en el recorrido que la construcción del profesional de enfermería ha tenido que sostener y que se perpetúa de alguna manera, con aires de innovación, en la formación de los futuros profesionales. La beneficencia, entendida como el "deber moral de obrar para beneficiar a otras personas" (Ferrer, 2012, p. 80), permanece latente en el espacio formativo, teñido del ímpetu por fortalecer la autonomía de las personas, de modo que el cuidado propio acompañe la libertad que todo ser humano, en distinta medida, busca para su vida. Como elementos centrales del cuidado se reconocen el orden, la disciplina y el control, que permiten disminuir cualquier posibilidad de incidente que signifique daño o desborde de alguna situación. Es frecuente que los profesionales de enfermería manifiesten que están "entrenados" para saber "todo" lo que sucede en su servicio, incluidos pacientes y personal a cargo. Entonces, nos preguntamos: ¿A qué mecanismos responden los enfermeros/as para brindar cuidados? ¿De qué manera están construidos los procesos que buscan mantener la vigilancia? Y por sobreto- 
do está la pregunta ¿Será posible superar esta perspectiva si se es hijo/a de ella?

Existen elementos de la historia de la enfermería que se mantienen y perfeccionan, haciendo posible una analogía partiendo desde lo que Florence Nightingale -considerada como la fundadora de la enfermería profesional- menciona en sus notas de enfermería del siglo XIX, en relación a la importancia de los pequeños detalles en la atención, que hacen la diferencia en la eficiencia que se logra, más allá de la abnegación y entrega del profesional:

"Todos los resultados de una buena enfermería, tal como se detallan en estas notas, pueden echarse a perder o ser completamente negativos, por un defecto, es decir: los pequeños detalles, o, en otras palabras, no saber conseguir que lo que usted hace cuando está allí se haga igual cuando no esté... la enfermera puede abandonar su salud y todos su otros deberes, y, sin embargo, por falta de un pequeño detalle, no ser la mitad de eficiente que otra que no es ni la mitad de entregada de ella, pero que tiene ese arte de multiplicarse a sí misma". (Nigthtingale, F. 1859. p. 30)

Lo anterior, da cuenta de la instalación de lógicas de control presentes en la atención directa de las personas, y la idea de que la eficiencia va de la mano del arte de multiplicarse a sí misma, de tal forma que las cosas ocurran aun cuando no esté el profesional, y el cuidado se perpetúe en ausencia del mismo; es decir mantener una vigilancia constante, que trasciende a la presencia de la persona. Así entonces, una vez que el dispositivo de vigilancia se instala, ya no es necesaria la presencia constante del enfermero/a.

Ahora bien, el cuidado de enfermería ha evolucionado en su conceptualización con el pasar de los años, siendo para Florence Nigthtingale al inicio de la enfermería moderna "un servicio a la humanidad basado en la observación y la experiencia, que consiste en poner a la persona enferma o sana en las mejores condiciones, a fin de que la naturaleza pueda restablecer o recuperar la salud" (Kérouack, 1996, p.48). Por su parte, una de las teóricas de enfermería más recientes, Jean Watson, define el cuidado como: "arte y ciencia humana del caring, ideal moral y procesos transpersonales dirigidos a la promoción de la armonía cuerpomuerte-espíritu utilizando diez factores de cuidado" (Kérouack, 1996, p. 56). Las perspectivas desde Nigthtingale a Watson evidencian la evolución que el pensamiento de enfermería ha tenido y sus esfuerzos por ampliarse, independizarse y adaptarse a los contextos.

En un intento por observar el cuidado desde la perspectiva de la biopolítica, parece pertinente considerar que "el cuidado tiene su fin en evitar la muerte" considerando que "ahora es en la vida y a lo largo de su desarrollo donde el poder establece su fuerza, la muerte es su límite" (...) "poder que se ejerce sobre la vida, el derecho individual y privado de morir..." (Foucault, 1977, p. 83). En este sentido, cuidamos para mantener la vida y de esta manera perpetuar el control sobre las personas, el cual acaba cuando finaliza la vida. Por su parte, la maquinaria tecnológica y humana, de la cual la enfermería también forma parte, está montada para hacer frente y vencer dicha muerte, como una manera de evitar la pérdida del control.

Existe un control social sobre el cuerpo de las personas, en donde se indica qué hacer, cómo cuidarse para evitar la muerte y de esa manera mantener una población productiva. Los pacientes sumisos son considerados buenos, tranquilos, agradables, sobre todo aquellos que nos "hacen caso". De tal modo, las estadísticas relacionadas con los parámetros de salud poblacionales, dan cuenta del 
éxito de las acciones que emprendemos como sociedad, en pro del sometimiento del cuerpo del otro y que cuenta con normas que lo respaldan. En este sentido, la enfermería se convierte en una efectiva herramienta de control, consagrado a él desde sus inicios en "disciplinar" sus integrantes y las personas a las que cuida. Las mismas lógicas de alguna manera han sido transferidas a la formación profesional de enfermeras, la cual desde sus inicios consideraba que la buena estudiante era aquella que "hacía lo que se le mandaba" (Donahue, 1985), "siendo evidente que la alumna que hacía demasiadas preguntas caía en la categoría de personalidad inapropiada para la enfermería", cuya consecuencia era la expulsión de la Escuela" (Castillo, 2007, p.2). Un par de siglos después de la instalación de las primeras escuelas de enfermería, se continúa evidenciando las múltiples formas en que el poder actúa en la formación de profesionales de enfermería, explicando los mecanismos mediante los cuales las ideologías dominantes dirigen a profesoras y estudiantes hacia la conformidad y subordinación, cuyas prácticas y lógicas serán replicadas en la posterior acción profesional en las instituciones sanitarias (Medina, 2006).

Por su parte, la enfermería con sus diversos sistemas de gestión del cuidado busca mantener y mejorar el nivel de salud de las personas, cuestión que se relaciona con la idea de que "las disciplinas funcionan cada vez más como unas técnicas que fabrican individuos útiles" denominado por Foucault como "la inversión funcional de las disciplinas" (Foucault, 2002, pp. 192-193). En consecuencia, se es un agente de biopoder desde la propia construcción profesional. Entonces, la genuina intención de ayuda al prójimo que moviliza el cuidado, se mezcla con la visualización de prevenir, pro-

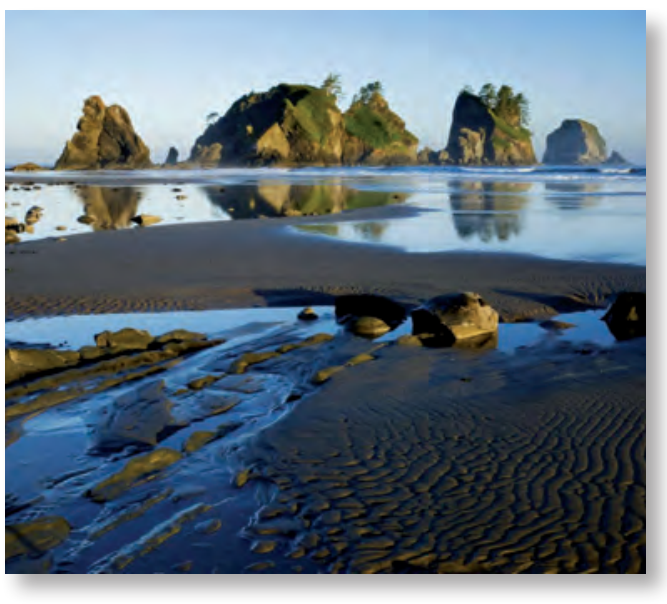

mover y recuperar la salud, para mantener individuos saludables durante el mayor tiempo posible, los cuales sean útiles al sistema social imperante.

Sumado a lo anterior, la revolución de la tecnología abre espacios de observación permanente en tiempo real. La presencia humana comienza a ser desplazada por dispositivos tecnológicos donde todo queda registrado, en el que la vida privada y la intimidad son espacios con límites cada vez más estrechos. Se ve de manera positiva para quienes requieren de cuidado, el estar permanentemente observados por dispositivos tecnológicos, sin presencia de el/la enfermero/a. De acuerdo a esto, se hace imprescindible "establecer una suerte de equilibrio reflexivo que permita asegurar que lo factible no prevalezca necesariamente por sobre lo legítimo" (Villarroel, 2013, p. 46), planteamiento que podría representar la era del panoptismo generalizado - entendido desde Bentham como "la figura arquitectónica de esta composición. Conocido es su principio: en la periferia, una construcción en forma de anillo; en el centro, una torre, ésta, con anchas ventanas que se abren en la cara interior del anillo. La construcción periférica está dividida en celdas, cada una de las cuales atraviesa toda la anchura de la construcción (...) De ahí el efecto 
mayor del Panóptico: inducir en el detenido un estado consciente y permanente de visibilidad que garantiza el funcionamiento automático del poder. Hacer que la vigilancia sea permanente en sus efectos incluso si es discontinua en su acción" (Foucault, 2002, p. 183).

\section{CONCLUSIONES}

La imagen de la enfermería y el cuidado como herramienta de control, instala tensiones en la reflexión vinculada al principio de beneficencia, considerada frecuentemente como una motivación primaria por cuidar a los más débiles. Lo anterior, hace necesario visualizar desde una perspectiva más amplia el propósito de nuestro quehacer disciplinar y profesional de enfermería, que abra la posibilidad de remirarnos y comprender que muchas veces nuestras prácticas desde lo cotidiano perpetúan lógicas de subordinación y poder.

Sólo el derrumbe de una estructura permite levantar otra, pero parece complejo siquiera darse la posibilidad de cuestionar lógicas disciplinares que se han construido durante siglos, y las consecuencias que éstas han tenido para las personas y la sociedad.

Sin embargo, el visibilizar estas cuestiones podría abrir espacio para que, conscientes del poder que constantemente es posible ejercer sobre las personas que son cuidadas, y para el cual se fue entrenado, pueda surgir una forma alternativa de concebir la enfermería. Buscar en los anales de la enfermería, nos daría la posibilidad de volver a pensar en valores intrínsecos de nuestra deontología profesional como son el respeto, la confiabilidad y trato digno, entre otros; de tal forma de construir saberes en torno al cuidado buscando la comprensión íntima de los procesos que pasa la persona a lo largo de su vida, respecto a los tránsitos entre salud y enfermedad.
Finalmente, se hace necesario que estas reflexiones sean parte de los procesos formativos de los futuros profesionales de enfermería y puedan hacerse parte también de las discusiones respecto las relaciones establecidas entre profesores y estudiantes, lo que sin duda requiera una transformación del paradigma disciplinar hasta ahora normalizado.

\section{BIBLIOGRAFÍA}

- Castillo, S., y Vessoni Guioti, R. (2007). La relación tutorestudiante en las prácticas clínicas y su influencia en el proceso formativo del estudiante de enfermería. Educare 21. 38 .

- Donahue, M.P. (1985). Historia de la enfermería. Barcelona: Doyma.

- Ferrer, J. (2012). La Bioética y sus Principios. En Beca, J.P. y Astete C. Eds, Bioética Clínica. Santiago: Mediterráneo.

- Foucault, M. (1977). Historia de la sexualidad, vol. I «La voluntad de saber", México D.F: Siglo XXI.

- Foucault, M. (2002). Vigilar y castigar. El nacimiento de la prisión. Buenos Aires: Siglo XXI Editores.

- Kérouack, S. (1996). El pensamiento enfermero. Barcelona: Elsevier.

- Medina, J. L. (1999) Pedagogía del cuidado: saberes y prácticas en la formación universitaria. Barcelona: Laertes.

- Medina, J.L. (2006). Deseo de cuidar y voluntad de poder. La enseñanza de la enfermería. Barcelona: Ediciones Universitat de Barcelona.

- Ministerio de Salud. Norma General Administrativa $N^{\circ}$ 19. Gestión del Cuidado de Enfermería para la Atención Cerrada, Exenta $\mathrm{N}^{\circ} 1127$.

- Nigthtingale, F. (1859). Notas de enfermería: qué es y qué no es. Barcelona: Editorial Masson S.A.

- Villarroel, R. (2013). Administración biopolítica de la intimidad en los Biobancos. Acta bioethica, 19(1), 39-47. 